\title{
Clinical factors associated with death in 3044 COVID-19 patients managed in internal medicine wards in Italy: results from the SIMI-COVID-19 study of the Italian Society of Internal Medicine (SIMI)
}

\author{
Elena Corradini ${ }^{1} \cdot$ Paolo Ventura ${ }^{1} \cdot$ Walter Ageno $^{2}$. Chiara Beatrice Cogliati ${ }^{3} \cdot$ Maria Lorenza Muiesan $^{4}$. \\ Domenico Girelli ${ }^{5}$. Mario Pirisi ${ }^{6}$. Antonio Gasbarrini ${ }^{7}$. Paolo Angeli ${ }^{8} \cdot$ Patrizia Rovere Querini $^{9} \cdot$ Emanuele Bosi $^{9}$. \\ Moreno Tresoldi ${ }^{9} \cdot$ Roberto Vettor $^{10} \cdot$ Marco Cattaneo $^{11} \cdot$ Fabio Piscaglia $^{12}$. Antonio Luca Brucato ${ }^{13}$. \\ Stefano Perlini ${ }^{14}$. Paolo Martelletti ${ }^{15}$. Roberto Pontremoli ${ }^{16}$. Massimo Porta ${ }^{17}$. Pietro Minuz ${ }^{18}$. Oliviero Olivieri ${ }^{19}$. \\ Giorgio Sesti ${ }^{20}$. Gianni Biolo ${ }^{21}$. Damiano Rizzoni ${ }^{22}$. Gaetano Serviddio ${ }^{23}$. Francesco Cipollone ${ }^{24}$. Davide Grassi ${ }^{25}$. \\ Roberto Manfredini ${ }^{26}$. Guido Luigi Moreo ${ }^{27}$. Antonello Pietrangelo ${ }^{1}{ }^{(\mathbb{D}} \cdot$ SIMI-COVID-19 Collaborators
}

Received: 19 February 2021 / Accepted: 9 April 2021 / Published online: 24 April 2021

(c) Società Italiana di Medicina Interna (SIMI) 2021

\begin{abstract}
During the COVID-19 2020 outbreak, a large body of data has been provided on general management and outcomes of hospitalized COVID-19 patients. Yet, relatively little is known on characteristics and outcome of patients managed in Internal Medicine Units (IMU). To address this gap, the Italian Society of Internal Medicine has conducted a nationwide cohort multicentre study on death outcome in adult COVID-19 patients admitted and managed in IMU. This study assessed 3044 COVID-19 patients at 41 referral hospitals across Italy from February 3rd to May 8th 2020. Demographics, comorbidities, organ dysfunction, treatment, and outcomes including death were assessed. During the study period, 697 patients (22.9\%) were transferred to intensive care units, and 351 died in IMU (death rate 14.9\%). At admission, factors independently associated with in-hospital mortality were age (OR 2.46, $p=0.000$ ), productive cough (OR 2.04, $p=0.000)$, pre-existing chronic heart failure (OR 1.58, $p=0.017$ ) and chronic obstructive pulmonary disease (OR 1.17, $p=0.048$ ), the number of comorbidities (OR 1.34, $p=0.000$ ) and polypharmacy (OR 1.20, $p=0.000$ ). Of note, up to $40 \%$ of elderly patients did not report fever at admission. Decreasing $\mathrm{PaO}_{2} / \mathrm{FiO}_{2}$ ratio at admission was strongly inversely associated with survival. The use of conventional oxygen supplementation increased with the number of pre-existing comorbidities, but it did not associate with better survival in patients with $\mathrm{PaO}_{2} / \mathrm{FiO}_{2}$ ratio $<100$. The latter, significantly benefited by the early use of non-invasive mechanical ventilation. Our study identified $\mathrm{PaO}_{2} / \mathrm{FiO}_{2}$ ratio at admission and comorbidity as the main alert signs to inform clinical decisions and resource allocation in non-critically ill COVID-19 patients admitted to IMU.
\end{abstract}

Keywords SARS-CoV-2 $\cdot$ Mortality from COVID-19 $\cdot$ Comorbidity $\cdot$ Polypharmacy $\cdot$ Internal medicine

\begin{tabular}{|c|c|c|}
\hline Abbr & & CKD \\
\hline $\mathrm{ACE}$ & Angiotensin-converting enzyme & COPD \\
\hline $\mathrm{CHF}$ & Chronic heart failure & COVID-19 \\
\hline & & CPAP \\
\hline Elena & nd Paolo Ventura contributed equally to the & $\mathrm{ICU}$ \\
\hline & & IMU \\
\hline Memb & IMI COVID-19 Collaborators are listed in the & LMWH \\
\hline Ackno & tt section at the end of the article. & NIV \\
\hline
\end{tabular}

Antonello Pietrangelo

antonello.pietrangelo@unimore.it

Extended author information available on the last page of the article
Chronic kidney disease

Chronic obstructive pulmonary disease

Coronavirus disease 2019

Continuous positive air pressure

Case report form

Intensive care units

Internal medicine units

Low molecular weight heparin

Non-invasive ventilation

Reverse-transcriptase polymerase chain reaction 


\section{$P / F \quad$ Arterial oxygen partial pressure $\left(\mathrm{PaO}_{2}\right.$ in $\mathrm{mmHg}) /$ fractional inspired oxygen $\left(\mathrm{FiO}_{2}\right.$ expressed as a fraction)}

SARS-CoV-2 Severe acute respiratory syndrome Coronavirus 2

\section{Introduction}

Since the beginning of 2020, the infection from SARS$\mathrm{CoV}-2$ has spread worldwide, and its clinical manifestation, the Coronavirus disease 2019 (COVID-19), has had dramatic consequences on global health [1]. As of December 24,2020 , there have been over 106,008,943 confirmed cases of COVID-19, including 2,316,389 deaths, reported to WHO (https://covid19.who.int).

Italy has been heavily hit during the COVID-19 2020 outbreak, in both the first and second pandemic phases [2-4]. Divisions of Infectious Disease, Pulmonology and Intensive Care Units were rapidly involved to face the very first pandemic phase, but the Internal Medicine wards were soon after called to the frontline [5, 6], reorganized and progressively expanded to eventually manage the large majority of hospitalized COVID-19 patients in Italy. A close collaboration between the Internists attending the Internal Medicine wards and the Emergency Departments was a key factor in the patients' flow within the Hospital and for finely tuning the admission and discharge criteria.

While a large body of data has been provided on characteristics and general management in hospitalized COVID-19 patients [7-10], relatively scarce information is available on clinical features and main outcomes in COVID-19 patients managed in Internal Medicine wards.

To address this knowledge gap, the Italian Society of Internal Medicine (SIMI) has conducted a national cohort multicenter study that examined demographics, comorbidities, organ dysfunction, treatment, and outcomes, such as length of hospital stay, admission to intensive care units (ICU), and death, in patients with COVID-19 admitted to and managed in Internal Medicine units (IMU) across Italy in February-May 2020.

\section{Methods}

\section{Study population and data collection}

A multicenter registry of adult patients hospitalized for COVID-19 in Internal Medicine wards was designed and promoted by the Italian Society of Internal Medicine (SIMI). We enrolled adults with a diagnosis of COVID-19 who were admitted to participating IMU at 41 large tertiary referral hospitals between February 3rd and May 8th 2020. As the registry recorded standard local practices, no specific treatments, tests, or procedures were mandated by the study protocol. All participating centers received approval from the local Ethics Committees.

Data were collected using electronic medical records and gathered in an anonymized case report form (CRF). The completeness and accuracy of data collected from the patient medical records were checked by the registry-coordinating center. We followed up patients until hospital discharge, death, or May 8th, 2020, whichever came first.

Inclusion criteria to the study were: age older than 18 years, and:

- detection of SARS-CoV-2 nucleic acid by reverse-transcriptase polymerase chain reaction (RT-PCR) test in nasopharyngeal swab/other biological specimens, or

- an epidemiological diagnosis of COVID-19, based on typical clinical features of SARS-CoV-2 infection (cough, fever, shortness of breath, sudden onset of anosmia/ageusia/dysgeusia) in association with

o a positive serological test for SARS-CoV-2 or

o features compatible with COVID-19 at chest imaging (computed tomography, ultrasonography or radiography)

For the purpose of this study, information on demographic variables (age and sex), medical history and laboratory data were collected. Data were manually curated to check for internal coherence (e.g., unit measures) and potential data entry errors. The $P / F$ ratio [i.e., the ratio of arterial oxygen partial pressure $\left(\mathrm{PaO}_{2}\right.$ in $\left.\mathrm{mmHg}\right)$ to fractional inspired oxygen $\left(\mathrm{FiO}_{2}\right.$ expressed as a fraction), also known as the Horowitz index] calculated at the hospital admission, was used to stratify the severity of pulmonary involvement in COVID-19 as light $(P / F \geq 300)$, mild $(P / F=299-200)$, moderate $(P / F=199-100)$ and severe $(P / F<100)$. The main outcome was in-hospital mortality (death).

\section{Statistical analyses}

Categorical variables are expressed as frequencies and percentage; Chi-square or Fisher exact test (when appropriated) were used in assessing the significance of difference of their prevalence between groups. Continuous variables are presented as mean and standard deviation, as appropriate, and were compared using the unpaired Student $t$ test when two groups were considered and ANOVA with Tukey's post hoc when more groups were considered; ANCOVA was used to compare continuous variables between groups in the presence of potential confounding factors.

As an approximation of the relative risk, odds ratio (OR) for each single considered risk factor was calculated by 
simple cross-tabulation with statistical significance evaluated by Chi-square test. Simple and multivariate logistic regression was used to assess the association between different considered variables and outcomes when dichotomous (death); simple and multivariate linear regression was used to assess the association between considered variables and outcomes, when continuous (length of hospital stay). In all statistical evaluations, a $p$ value $<0.05$ was considered as significant. Statistical analysis were conducted using SPSS ${ }^{\circledR}$ (SPSS v.25.0, Chicago, Il, USA) and STATA ${ }^{\circledR}$, (STATA version 15.0, College Station, TX, USA) software.

\section{Results}

A total of 3170 patients hospitalized in IMU for COVID19 were enrolled. After quality check and elimination of 126 records for incompleteness, data of 3044 out of 3170 patients $(96 \%)$ were included in the study [mean (SD) age 67 (15) years; 1961 (64\%) male]. Detection of SARS-CoV-2 RNA in nasopharyngeal swab by RT-PCR test was positive in $95.8 \%$ of patients.

Symptoms recorded at disease presentation as percentage and according to age (decades) are reported in
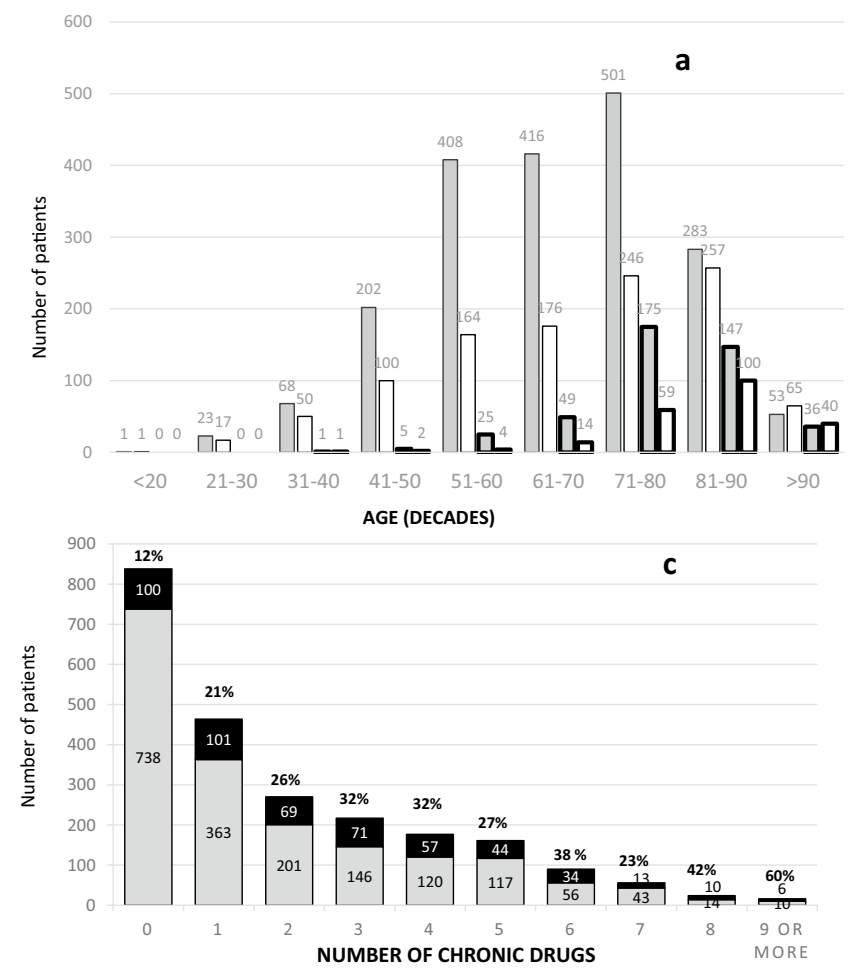

Fig. 1 Panel a shows the distribution of male (gray columns) and female (white columns) patients admitted to IMU and of deceased patients (black framed columns) by age decades. The distribution of deceased (black columns) and surviving (gray columns) patients stratified by number of comorbidities or chronic drugs is shown in
Supplementary Table 1. Main symptoms were fever (85.8\%), productive cough (44.4\%), pharyngodynia (42.2\%), asthenia (21\%) and dry cough (20.5\%).

During the study period, 661 patients died (21.7\%). Out of the 697 patients (22.9\%) who had been transferred from IMU to ICU, 310 died, while the remaining 351 deaths occurred in IMU. Figure 1a reports the distribution of patients by sex and age, and the fraction of deceased patients. The mortality rate, generally higher in males than in females, increased steadily in patients older than 70 years: from $31.3 \%$ in the $71-80$ decade to $47.5 \%$ in the $81-90$ decade, and $64.4 \%$ in patients older than 90 years. Death rate was significantly higher in males than in females in the $71-80$ and in the 81-90 decades $(p=0.0025$ and $p=0.0034$, respectively), while an opposite but not significant trend was found in patients older than 90 years.

\section{Data and analyses at hospital admission}

Mean age at admission was significantly higher in deceased patients compared to patients eventually discharged from the hospital ( $79 \pm 10$ vs. $64 \pm 15$ years, $p=0.000)$, and predicted a higher risk for in-hospital mortality (OR for age decade $=2.46,95 \%$ CI 2.2-2.7, $p=0.000$ ). Symptoms more
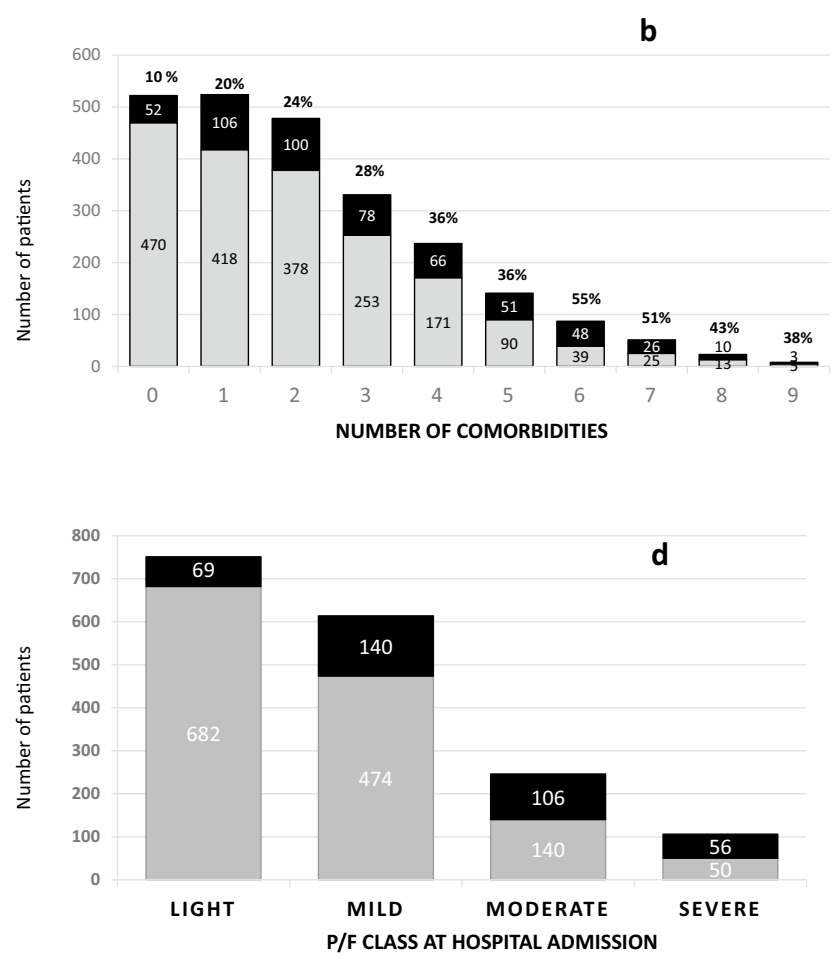

panel $\mathbf{b}$ and $\mathbf{c}$, respectively (the percentages above the black columns refer to the mortality rate). Panel $\mathbf{d}$ shows the distribution of deceased (black columns) and surviving (gray columns) patients by $P / F$ class at admission 
frequently associated with mortality at multivariate analysis were fever (OR 0.602, 95\% CI 0.402-0.903, $p=0.014$ ), productive cough (OR 2.04, 95\% CI 1.51-2.77, $p=0.000$ ) pharyngodynia (OR $0.343,95 \%$ CI $0.245-0.478, p=0.000$ ), diarrhea (OR 0.563, 95\% CI 0.333-0.952, $p=0.031$ ), anos$\mathrm{mia} /$ dysgeusia (OR $0.240,95 \%$ CI $0.072-0.805, p=0.020$ ), dry cough (OR $0.578,95 \%$ CI $0.376-0.890, p=0.013$ ) and muscle pain (OR $0.356,95 \%$ CI $0.156-0.818, p=0.020$ ) (Table 1).

When considering the frequency of pre-existing medical conditions, a significant association with death was found as crude OR (univariate analysis) for cardiovascular disease, chronic heart failure (CHF), atrial fibrillation, hyperlipidemia, chronic kidney disease (CKD), and dementia (Table 2). However, after multivariate analysis, only CHF and COPD were significantly associated to exitus. Figure $1 \mathrm{~b}$ reports patients' outcome (number of discharged and deceased patients) and mortality rate according to the number of comorbid conditions at admission. A significant association between number of comorbidities and death was observed, even after stratification for disease severity $(P / F$ classes) (OR 1.34, 95\% CI 1.28-1.44, $p=0.000$ ).

The number of reported chronic medications was $2.33 \pm 2.3$ per patient, and significantly increased with the severity of pulmonary involvement $(1.89 \pm 2.2$ vs. $2.6 \pm 2.1$, vs. $2.7 \pm 2.4$, vs. $3.0 \pm 2.2$, from $P / F$ class 1 to class $4, p$ for trend $=0.000$ ). Most frequently reported drugs were beta-blockers (23.5\%), ACE inhibitors (21.4\%), acetylsalicylic acid (20.4\%), diuretics (19.2\%), statins
(18.3\%) and angiotensin II receptor blockers (17.6\%) (Table 3). Figure 1c reports patients' outcome (number of discharged or deceased patients) and mortality rate according to the number of chronic medications. A significant association was observed between the number of drugs and death (OR 1.20, 95\% CI 1.15-1.25, $p=0.000)$.

Demographics and biochemical parameters at hospital admission and patient stratification according to $P / F$ classes are reported in Supplementary Table 2. Most variables show a significant difference across worsening $P / F$ classes, but only $P / F$ class remained an independent predictor of death after multivariate analysis (Supplementary Table 3). In fact, mortality rate significantly increased in patients with more severe pulmonary involvement (Fig. 1d): from $9.2 \%$ in light to $52.8 \%$ in severe $P / F$ class ( $p$ for trend $=0.000$ ). The $P / F$ classes were strongly inversely associated with survival (OR $0.419,95 \%$ CI $0.367-0.477, p=0.000$ ).

Figure 2 reports the distribution of admitted patients over time (weeks), stratified according to $P / F$ classes and mortality rate. Of note, patients who died in IMU were older ( $81 \pm 9$ vs. $77 \pm 10$ years, $p=0.000$ ), presented a higher number of comorbidities $(3.6 \pm 2.2$ vs. $2.3 \pm 1.9, p=0.000)$ and reported a higher number of medications $(3.1 \pm 2.2 \mathrm{vs}$. $1.9 \pm 1.2 p=0.000)$ than those dying after transfer to ICU. On the other hand, at admission, the degree of severity of pulmonary involvement (light, mild, moderate, or severe based on $P / F$ ratio) was not significantly different between patients who died in IMU and those deceased after transfer
Table 1 Main symptoms and their association with mortality

\begin{tabular}{lllll}
\hline & $\begin{array}{l}\text { OR }(95 \% \text { CI }) \\
\text { (unadjusted) }\end{array}$ & $p$ & $\begin{array}{l}\text { OR }(95 \% \text { CI }) \\
\text { (adjusted) }\end{array}$ & $p$ \\
\hline Fever $(n=2085)$ & $.494(.371-.657)$ & .000 & $.602(.402-.903)$ & .014 \\
Headache $(n=1684)$ & $.224(.081-.610)$ & .004 & & \\
Abdominal pain $(n=1380)$ & $1.04(.283-3.78)$ & .957 & & \\
Conjunctivitis $(n=1629)$ & $1.25(.400-3.89)$ & .703 & & \\
Rhinorrea $(n=1673)$ & $1.11(.475-2.61)$ & .804 & & .000 \\
Pharyngodynia $(n=1354)$ & $.414(.306-.558)$ & .000 & $.343(.245-.478)$ & .013 \\
Dry cough $(n=1437)$ & $.676(.478-.937)$ & .027 & $.578(.376-.890)$ & .000 \\
Productive cough $(n=1945)$ & $2.13(1.69-2.68)$ & .000 & $2.04(1.51-2.77)$ & \\
Dyspnea $(n=1724)$ & $1.20(.889-1.62)$ & .231 & & .031 \\
Vomiting $(n=1885)$ & $.583(.313-1.08)$ & .088 & & .014 \\
Diarrhea $(n=1877)$ & $.382(.243-.603)$ & .000 & $.563(.333-.950)$ & \\
Muscle pain $(n=1682)$ & $.271(0.124-0.581)$ & .001 & $.356(.156-.818)$ & \\
Asthenia $(n=1631)$ & $.790(.578-1.08)$ & .141 & & .021 \\
Anosmia/dysgeusia $(n=1952)$ & $.232(.094-.577)$ & .002 & $.240(.072-.805)$ & \\
Syncope $(n=1902)$ & $.979(.538-1.78)$ & .946 & & \\
Cutaneous signs $(n=1597)$ & $.318(.041-2.46)$ & .273 & & \\
\hline
\end{tabular}

Simple and multiple logistic regression was used to assess, respectively, univariate (unadjusted) and independent (adjusted) associations between symptoms and in-hospital mortality; variables with a $p$ value $<0.1$ at simple logistic regression (unadjusted) were included in the multiple logistic regression model

$O R$ odds ratio, $C I$ confidence interval. 
Table 2 Main comorbidities and their association with mortality
Table 3 Chronic medications and their association with mortality

\begin{tabular}{llllll}
\hline & \% of patients & $\begin{array}{l}\text { OR }(95 \% \text { CI }) \\
\text { (unadjusted) }\end{array}$ & $p$ & $\begin{array}{l}\text { OR (95\% CI) } \\
\text { (adjusted) }\end{array}$ & $p$ \\
\hline Hypertension & 48.8 & $.853(.689-1.06)$ & .147 & \\
Cerebrovascular disease & 11.8 & $1.32(.972-1.81)$ & .074 & \\
Cardiovascular disease & 14.05 & $1.35(1.04-1.76)$ & .026 & & \\
Chronic heart failure & 17.1 & $1.56(1.27-1.98)$ & .014 & $1.58(1.15-1.95)$ & .017 \\
Atrial fibrillation & 11.1 & $1.51(1.07-2.14)$ & .016 & \\
Diabetes & 21.6 & $1.01(.787-1.32)$ & .882 & \\
Hyperlipidemia & 17.4 & $1.33(1.01-1.77)$ & .046 & \\
COPD & 10.3 & $1.23(.964-1.76)$ & .081 & $1.17(1.04-1.98)$ & .048 \\
Asthma & 3.8 & $.895(.471-1.70)$ & .736 & & \\
Pulmonary fibrosis & 0.9 & $.871(.244-3.10)$ & .834 & \\
Chronic liver disease & 3.4 & $1.98(.651-5.96)$ & .213 & \\
Inflammatory bowel disease & 1.1 & $1.06(.345-3.26)$ & .903 & \\
Chronic kidney disease & 29.9 & $1.30(1.08-1.69)$ & .043 & \\
Cancer & 13.2 & $1.21(.900-1.64)$ & .197 & \\
Dementia & 11.8 & $1.90(1.38-2.63)$ & .000 & \\
Depression & 5.7 & $1.07(.681-1.68)$ & .764 & \\
Hypothyroidism & 8.1 & $1.02(.651-1.58)$ & .914 & \\
\hline
\end{tabular}

Simple and multiple logistic regression was used to assess, respectively, univariate (unadjusted) and independent (adjusted) associations between comorbidities and in-hospital mortality; variables with a $p$ value $<0.1$ at simple logistic regression (unadjusted) were included in the multiple logistic regression model

$O R$ odds ratio, $C I$ confidence interval

${ }^{\text {a }}$ Data available in 1505 patients

\begin{tabular}{lccc}
\hline & \% of patients $^{\mathrm{a}}$ & $\begin{array}{l}\text { OR }(95 \% \text { CI }) \\
\text { (unadjusted) }\end{array}$ & $p$ \\
\hline Ace-inhibitors & 21.4 & $1.23(.953-1.57)$ & .111 \\
Angiotensin II receptor blockers & 17.6 & $1.16(.897-1.49)$ & .261 \\
Beta-blockers & 23.5 & $1.28(.998-1.67)$ & .052 \\
Ca-antagonists & 15.8 & $1.11(.816-1.51)$ & .507 \\
Metformin & 9.9 & $1.24(.837-1.78)$ & .252 \\
Insulin & 6.1 & $1.09(.688-1.69)$ & .734 \\
Anti-aldosterone agents & 6.2 & $1.64(.765-1.76)$ & .477 \\
Diuretics & 19.2 & $1.40(1.08-1.80)$ & .009 \\
Statin & 18.3 & $1.06(.817-1.37)$ & .658 \\
DOAC & 6.1 & $1.19(.759-1.89)$ & .437 \\
AVK & 4.2 & $1.36(.806-2.29)$ & .248 \\
Acetyilsalicylic acid & 20.4 & $1.57(1.18-2.08)$ & .002 \\
Inhaled corticosteroids & 4.4 & $.868(.448-1.62)$ & .629 \\
SABA/LABA & 4.8 & $.853(.356-1.33)$ & .274 \\
LAMA & 2.6 & $1.72(.850-3.48)$ & .131 \\
Immunosuppressive drugs & 5.4 & $1.14(.987-1.77)$ & .079 \\
\hline
\end{tabular}

Simple logistic regression was used to evaluate the associations between chronic drugs and in-hospital mortality

$D O A C$ direct oral anticoagulant, $A V K$ anti-vitamin $\mathrm{K}, S A B A$ short-acting $\beta 2$-agonist, $L A B A$ long-acting $\beta 2$-agonist, LAMA long-acting muscarinic antagonist, $O R$ odds ratio, $C I$ confidence interval

${ }^{a}$ Data available in 2219 patients 


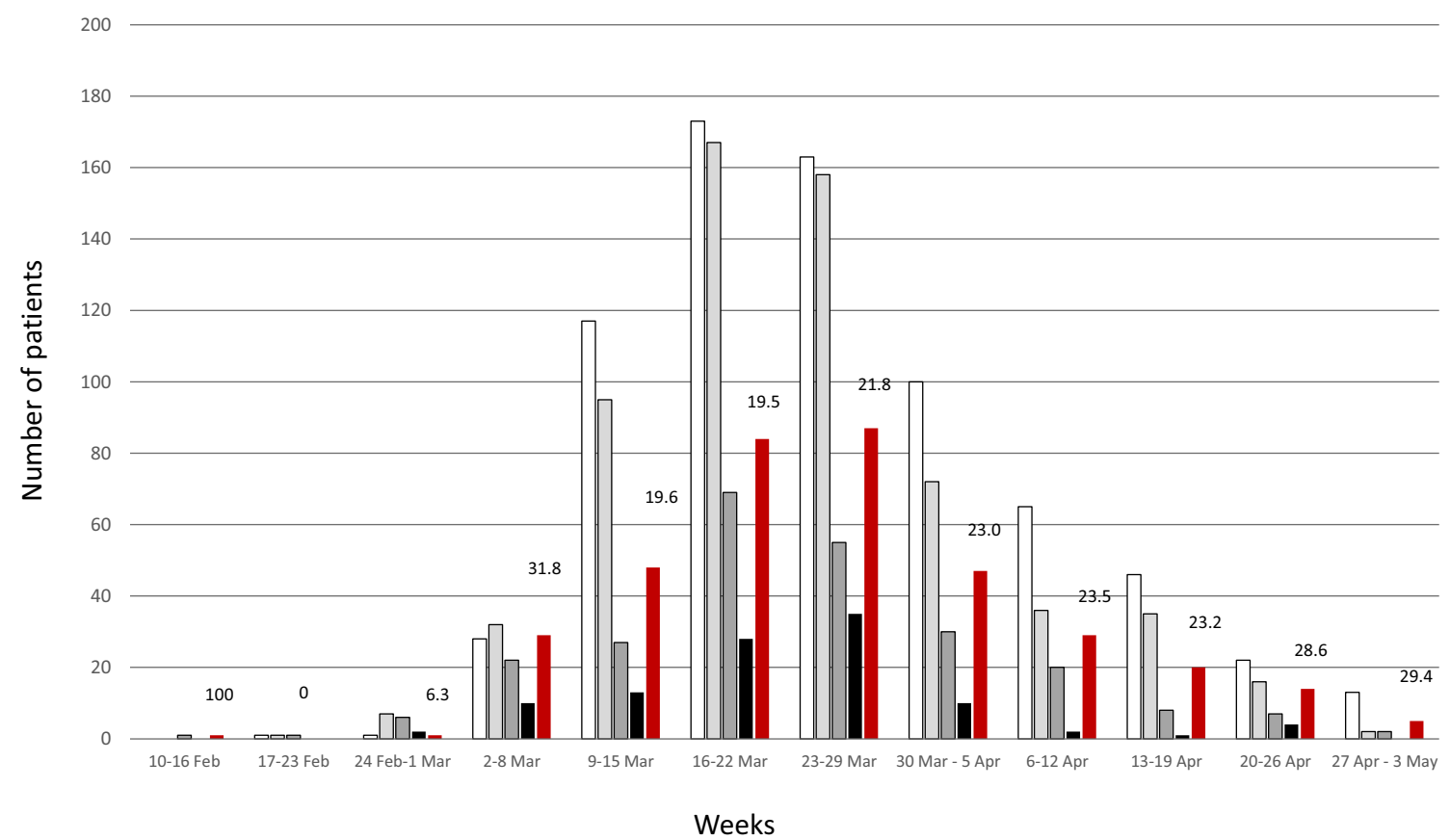

Fig. 2 The distribution of admitted patients over time (weeks) is stratified according to $P / F$ classes and the mortality rate. The severity of respiratory impairment is represented in grayscale from light (white

in ICU $(12.4 \%, 18.6 \%, 30.6 \%, 38.2 \%$ vs. $14.4 \%, 19.7 \%$, $35.6 \%, 43.8 \%$, respectively; $p=0.645$ ) (data not shown).

\section{Data and analyses during hospital stay}

During hospital stay, the mortality rate significantly increased in patients with worsening pulmonary involvement (Fig. 1d). Interestingly, the highest mortality rate (nearly $32 \%$ ) was recorded at the pandemic burst [Fig. 2, week 4 (March 2-4, 2020)] when patients more frequently belonged to the moderate-to-severe $P / F$ classes and had a higher number of pre-existing comorbidities $(69.5 \%$ vs $52.3 \%, p=0.001$ and $2.76 \pm 1.8$ vs. $2.37 \pm 1.9, p=0.067$, respectively).

COVID-19-related drugs used during hospital stay have been stratified according to disease severity (Supplementary Table 4) and outcome (survival/death) (Supplementary Fig. S1). Of note, in IMU 84\% of patients used hydroxychloroquine and $80.7 \%$ received oxygen supplementation (Supplementary Table 4). In addition, a significant trend in the rate of use of medications was observed across worsening $P / F$ classes.

An association study between the use of COVID-19-related medications and death by univariate analysis (Supplementary Table 5) and multivariate analysis (Supplementary Table 6) was performed in 2209 patients fully managed in IMU until discharge or death. Data are reported both as cumulative and stratified according to the degree of disease severity ( $P / F$ classes). At the multivariate analysis, the use columns) to severe (black columns) $P / F$ class. The red columns show the number of deceased patients (the numbers above the columns refers to the mortality rate)

of hydroxychloroquine was significantly associated with survival in light, mild and moderate classes of severity of $P / F$ dysfunction (Supplementary Table 6), the use of antivirals was associated with survival in mild and moderate $P / F$ classes, while LMWH and tocilizumab were significantly associated with progressive better survival from the mild to the severe classes as assessed according to the odds ratios, becoming statistically significant for benefit in the more severe $P / F$ classes. Intravenous steroids did not prove to be beneficial in any class.

As to oxygen supplementation, $78.1 \%$ of 2409 patients in whom data were available were treated with oxygen therapy (mean age $69 \pm 14$ ) while $21.9 \%$ did not receive oxygen supplementation (mean age $62 \pm 13$ ). Patients treated with oxygen supplementation were significantly older than those untreated $(p<0.01)$.

When analyzing the different oxygen delivery systems, $71.8 \%$ of 1578 patients in whom data were available received conventional supplemental oxygen therapy (i.e., nasal cannulae, simple face mask, ventimask, reservoir) (mean age $70 \pm 15$ ), and $23.5 \%$ received non-invasive mechanical ventilation [Continuous Positive Air Pressure (CPAP), NonInvasive Ventilation (NIV)] (mean age $67 \pm 14$ ). Figure 3a reports the use of conventional oxygen supplementation and non-invasive mechanical ventilation during hospital stay by age. The percentage of patients who received conventional oxygen supplementation increased with the number of comorbidities: from about $60 \%$ in non-comorbid patients 
Fig. 3 The percentage of patients treated by conventional oxygen supplementation (gray columns) and non-invasive mechanical ventilation (black columns) stratified by age decades (panel a) or number of comorbidities (panel b) is shown

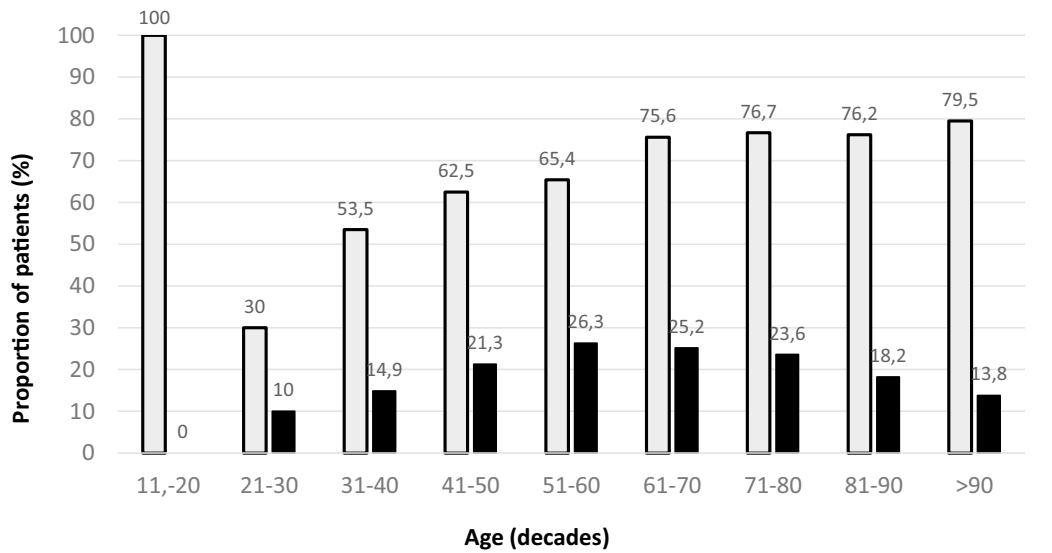

a

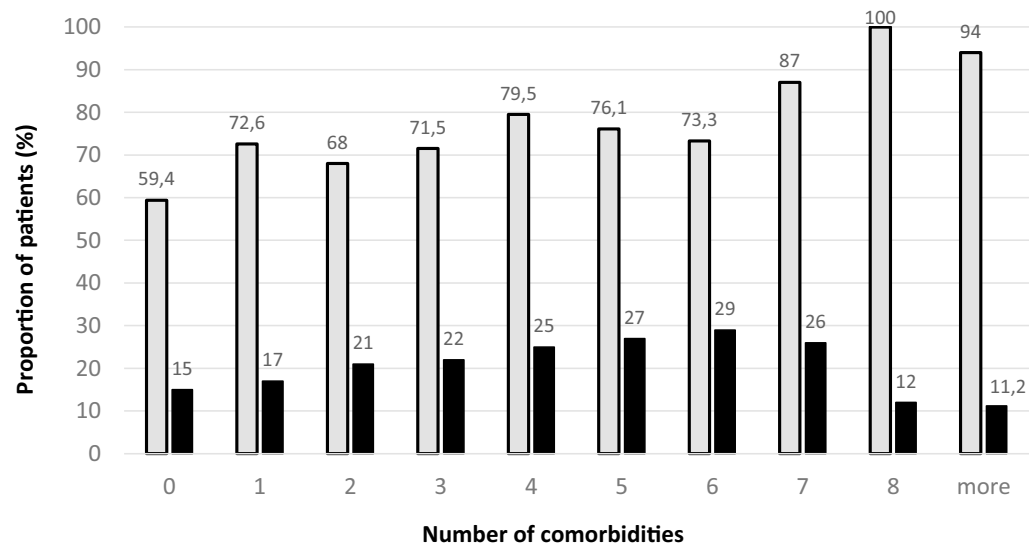

to about $90-100 \%$ in patients with more than six comorbidities (Fig. 3b). Of note, only nearly $11 \%$ of patients with more than 7 comorbidities were treated with non-invasive mechanical ventilation (Fig. 3b). In Supplementary Table 7, results of the association analysis between conventional oxygen supplementation or non-invasive mechanical ventilation and death are reported both as cumulative and according to the degree of disease severity ( $P / F$ classes). When considering the severity of pulmonary involvement at the time of admission, conventional oxygen supplementation was associated with lower mortality rate in patients in the light (OR 0.630, 95\% CI 0.393-1.0, $p=0.054$ ), mild (OR 0.233, 95\% CI 0.081-0.471, $p=0.007$ ) and moderate (OR 0.577, 95\% CI 0.265-1.02, $p=0.059) P / F$ classes, whereas noninvasive mechanical ventilation was associated with lower mortality in patients in moderate-to-severe $P / F$ classes (OR $0.377,95 \%$ CI $0.265-0.635, p=0.015$ and OR $0.235,95 \%$ CI 0.056-0.365, $p=0.009$, respectively).

Mean duration of hospitalization for discharged patients was $14.6 \pm 12.3$ days. Length of hospital stay (days) was significantly associated with patient age $(b=1.422,95 \%$ CI $1.05-1.791, p=0.000)$ and severity of pulmonary involvement (measured as $P / F$ classes) $(b=1.641,95 \% \mathrm{CI}$ $0.981-2.301, p=0.000)$. Patients discharged after transfer to ICUs had a longer hospital stay than those discharged from
IMUs $(18.7 \pm 18.1$ vs. $13.3 \pm 11.3$ days, $p=0.000)$, even after adjusting for disease severity.

\section{Discussion}

SARS-CoV-2 pandemic has subjected national health systems and hospitals to an unprecedented pressure all over the world, both during the first and the second 2020 COVID-19 pandemic waves.

We report here the results of the first multicentre, observational, nationwide study on death outcome in a large cohort of COVID-19 patients admitted and managed in IMU. Data have been recorded during the first pandemic surge in 41 large tertiary referral hospitals, when Italy reported the world second largest number of COVID-19 cases after China, and a remarkable case-fatality rate (https://www.who. int/docs/default-source/coronaviruse/situation-reports/20200 401-sitrep-72-covid-19.pdf?sfvrsn=3dd8971b_2).

The cumulative death rate in this study was $21.7 \%$, thus indicating the high lethality rate of SARS-CoV-2 infection in hospitalized patients. A similar mortality rate (from 20 to $26 \%$ ) has been reported in other large national and international studies [8,10-12]. The highest lethality rate was observed in patients older than 70 years, and generally in 
men vs women except for patients $>90$ years (Fig. 1a). This may be due to the fact that men in the 70-80 years decade had a more severe pulmonary involvement at admission (prevalence of moderate-to-severe $P / F$ class at admission were $66.4 \%$ in men vs. $52.8 \%$ in female, $p=0.037$ ) and in the $81-90$ years decade were slightly older than women $(85 \pm 3$ vs. $82 \pm 2 p=0.01$ ), reported a higher number of comorbidities ( $3.32 \pm 1.1$ vs. $2.98 \pm 1.2 p=0.052)$ and chronic medications $(3.26 \pm 2.3$ vs. $2.85 \pm 1.85, p=0.037)$.

Predicting the clinical trajectory of individual patients hospitalized with COVID-19 is extremely important and necessary to direct clinical care.

In the cohort of COVID-19 patients described in the present study, the most important predictor of death at admission was the $\mathrm{PaO}_{2} / \mathrm{FiO}_{2}$ ratio. In fact, while demographics and biochemical parameters, including age, were directly associated with worsening $P / F$ classes (Supplementary Table 2) and severe outcome (Supplementary Table 3), at multiple logistic regression analysis only the $P / F$ classes were strong and independent predictors of death (Supplementary Table 3). Recently, prediction models for death or severe disease in COVID-19 patients have been proposed [13, 14]: the COVID-19 Inpatient Risk Calculator (CIRC), based on factors present on admission, including age, age interaction with nursing home, Charlson Comorbidity Index (CCI), and $\mathrm{SaO}_{2} / \mathrm{FiO}_{2}$ ratio [13]; the COVID-19 adaptive risk predictor (SCARP), an interactive and dynamic tool providing 1-day and 7-day risk predictions on the basis of clinical information, including $\mathrm{SaO}_{2} / \mathrm{FiO}_{2}$ [14]. Although differences exist between our study and these studies, such as ethnicity and demographics of patient populations, the use of $\mathrm{PaO}_{2}$ instead of $\mathrm{SaO}_{2}$ and a binary outcome (death $\mathrm{Y} / \mathrm{N}$ ) instead of time to death or severe illness, they all agree on the fact that an early assessment of the respiratory impairment through $\mathrm{PaO}_{2}$ or $\mathrm{SaO}_{2}$ is crucial at identifying disease severity.

The number of comorbidities per se was also a strong predictor of death, even after stratification for disease severity (Table 2 and Fig. 1b). Interestingly, while nearly half of the individual chronic disorders appeared to negatively affect survival, only CHF and COPD were independently associated with death (Table 2). Accordingly, also pre-existing polypharmacy was associated with negative disease course and exitus in COVID-19 patients (Fig. 1c), whereas drugs used in CHF (diuretics and beta-blockers) or cardiovascular disease (aspirin) treatment were significantly associated with death, supporting the notion of a negative impact of cardiovascular comorbidities.

Of note, the highest mortality rate was recorded at the pandemic burst (Fig. 2), when patients more frequently belonged to the moderate and severe $P / F$ classes and reported a higher number of chronic diseases, reinforcing the concept that respiratory involvement on admission and comorbidity burden should guide the physician's alert and clinical decisions.

When assessing the prediction power of clinical symptoms at presentation, only productive cough, which suggests lower respiratory tract involvement and/or higher likelihood of bacterial superinfection, was independently associated with death, while fever, the most frequent symptom at presentation, was negatively associated with death (Table 1). This may indicate that patients able to mount an adequate systemic inflammatory response may have a less severe disease course. Interestingly, $30 \%$ of patients over 80 years and $40 \%$ over 90 years did not report fever at presentation, thus indicating the need for high level of COVID-19 clinical suspicion in the elderly, even if afebrile (Supplementary Table 1).

When considering laboratory data, CRP, ferritin and white blood cell count were positively associated with patient exitus, while hemoglobin, red blood cell and lymphocyte count were negatively associated to death. Only $P / F$ class was independently associated with death after multivariate analysis (Supplementary Table 3).

Dry cough, probably due to prevailing pharyngeal and large airways involvement, was common and was negatively associated with death. Its prevalence was higher in patients on chronic ACE inhibitors than in untreated patients [(74/299 (24.7\%) vs 194/1074 (18.1\%), $p=0.01$; Chi-square test]. Yet, one out of five untreated patients in our cohort reported this symptom. Dry cough might be due to irritation of cough receptor by SARS-CoV-2 infection and compensatory host ACE inhibitory response, further worsened by pharmacological ACE inhibition, as suggested [15]. On the other hand, although the influence of SARS-CoV-2 infection on the renin-angiotensin system enzymatic cascade is still a matter of active debate and research $[15,16]$, human studies have not reported higher SARS-CoV-2 infection risk or COVID-19 severity in ACE inhibitors users to date [15-17]. In fact, chronic therapy with ACE inhibitors was not associated with mortality in our COVID-19 cohort.

During hospital stay in IMU, different COVID-19-related drugs were used (Supplementary Table 4 and Supplementary Fig. S1), similarly to other reports in a comparable patient population and settings [18]. The use of hydroxychloroquine was significantly associated with survival in lightto-moderate $P / F$ classes, while antivirals were associated to survival in mild-to-moderate $P / F$ classes. Of note, LMWH and tocilizumab were significantly associated with survival only in more severe $P / F$ classes (i.e., severe and moderate, respectively) (Supplementary Table 6). The interpretation of the results on COVID-19 related drugs is limited by the observational nature of the study, the lack of specific analyses by dosages, routes and timing of drug administration, and local protocols and recommendations that varied over 
time across participating IMU. These limits, including the fact that systemic steroids administration was discouraged early in the pandemic, may likely concur to explain the lack of a beneficial effect of steroid therapy on survival in any $P / F$ class in our study population, in contrast with recent studies [19]. Despite these drawbacks, it is worth noting that either intravenous or subcutaneous tocilizumab administration was associated with a reduced risk of death in patients with 100-200 $P / F$ at the time of admission in agreement with observational reports [9]. Interestingly, a recent RCT on tocilizumab in hospitalized patients with COVID-19 pneumonia reported a reduction in the likelihood of progression to the composite outcome of mechanical ventilation or death, although no improvement in overall survival was observed [20].

Conventional supplemental oxygen therapy and noninvasive mechanical ventilation (CPAP, NIV) were used in a large proportion of patients managed in IMUs $(71.8 \%$ and $23.5 \%$, respectively). Conventional oxygen supplementation was associated with higher survival rate in light-tomild classes, whereas non-invasive mechanical ventilation was associated with improved survival in moderate-to-severe $P / F$ classes, indicating the need for early stratification of the degree of respiratory impairment to promptly choose and setup the appropriate non-invasive oxygen supplementation strategy. Of note, in agreement with significant relationship between the comorbidity burden and death (Fig. 3b), the number of patients requiring conventional oxygen therapy increased in parallel with the number of comorbidities.

As expected, when the comorbidity burden exceeded a critical level (i.e., more than seven comorbidities, Fig. 3b), a significantly lower percent of patients was assisted with noninvasive mechanical ventilation likely due to clinical prediction of non-beneficial treatment in highly comorbid elderly patients. Non-invasive oxygenation strategies were found to be associated with a lower risk of death when compared with standard oxygen therapy in a meta-analysis [21]. However, the mean age in the selected studies was lower than in the present cohort, and likely the comorbidity burden. This suggests that IMUs have managed older COVID-19 patients with more comorbidities, as also observed outside of this pandemic, and explains the high mortality rates reported. In a recent multicentric study addressing non-invasive ventilation out of ICU, the presence of "Do Not Intubate decision" was associated with a threefold risk of NIV failure. Moreover, $78 \%$ of patients who died in non-ICU wards had a "Do Not Intubate decision" [22], confirming that pre-existing conditions (age, comorbidity, frailty) have a major impact both in non-invasive ventilation success and in patient's prognosis [22, 23]. In keeping with this, when comparing patients dying in IMU and after transfer to ICU, although the severity of pulmonary involvement was not different between the two groups at admission, patients who died in
IMU were older, with a higher number of comorbidities and chronic medications.

When interpreting data on oxygen supplementation and ICU transfer, it should be considered that the decision-making on oxygen delivery systems, oxygenation goals, timing and type of non-invasive mechanical ventilation as well as ICU transfer were not pre-established among participating centers, and were mostly guided by local expertise and resource availability.

When considering all patients discharged from the hospital, length of hospital stay (days) was significantly associated with patient age, whereas patients discharged after transfer to ICUs had a longer hospital stay than those discharged from IMU, even after adjusting for disease severity, possibly due to the weaning from mechanical ventilation and complications connected to the advanced and invasive ICU therapies. In general, this study also confirms the key role of Internal Medicine in patient management and hospital flow also in the pandemic setting. Stratifying prognosis upon patient's admission allows a better treatment and allocation strategy, optimizing hospital resources [24].

In conclusion, in this multicentre cohort study of patients with COVID-19 admitted to IMU, we found that 1 in 5 was transferred to ICU and nearly 1 in 6 died, the majority required conventional oxygen supplementation and 1 out of 4 received non-invasive mechanical ventilation. We identified a number of risk factors at admission associated with death, such as age, specific symptoms such as productive cough, multi-morbidity and polypharmacy. The strongest predictors of death at admission were the $\mathrm{PaO}_{2} / \mathrm{FiO}_{2}$ ratio and the number of comorbidities. The use of conventional oxygen supplementation increased with the number of pre-existing comorbidities and improved survival of patients with mild pulmonary involvement, but it did not associate with better survival in patients with low $\mathrm{PaO}_{2} / \mathrm{FiO}_{2}$ ratio who benefited of early non-mechanical ventilation. During the first pandemic wave, standardized protocols of Covid-19 patient management and treatment were lacking, while non-homogeneous strategies based on local expertise and resources were often in place. Moreover, a number of implemented therapeutic strategies have now been superseded by different or modified approaches. For all these reasons, caution should be used when drawing recommendations in studies performed during the first pandemic wave, particularly when assessing the use of COVID-19-related drugs and severe outcomes. All this notwithstanding, we believe that this report provides a useful guide at identifying most critical alert signs and better approach for management of Covid-19 patients admitted and cared for in IMU wards.

Supplementary Information The online version contains supplementary material available at https://doi.org/10.1007/s11739-021-02742-8. 
Acknowledgements Members of the SIMI COVID-19 Collaborators: Elisabetta Tombolini, Department of Medicine and Surgery, University of Insubria, Varese, Italy; Thomas Teatini, Department of Internal Medicine, Luigi Sacco Hospital, University of Milan, Milan, Italy; Massimo Salvetti, Dept of Clinical \& Experimental Sciences, University of Brescia-Medicina 2, ASST Spedali Civili Brescia, Brescia, Italy; Ernesto Crisafulli, Department of Medicine, Section of Internal Medicine, University of Verona, Verona, Italy; Pier Paolo Sainaghi, Internal Medicine Department of Translational Medicine, University of Piemonte Orientale, Novara, Italy; Lorenzo Zileri Dal Verme, Department of Medical and Surgical Sciences. Fondazione Policlinico Gemelli IRCCS, Università Cattolica del S. Cuore, Rome, Italy; Salvatore Piano, Unit of Internal Medicine and Hepatology (UIMH), Department of Medicine-DIMED, University of Padova, Padova, Italy; Rebecca De Lorenzo, Unit of Internal Medicine, IRCCS Ospedale San Raffaele, Vita-Salute San Raffaele University, Milan, Italy; Gaetano Arcidiacono, Department of Medicine, Università degli Studi di Padova, Padova, Italy; Marco Podda, Dipartimento di Scienze della Salute, Università degli Studi di Milano, Milan, Italy; Luca Muratori, Unit of Internal Medicine, Azienda Ospedaliero Universitaria S.Orsola Malpighi, Bologna, Italy; Claudia Gabiati, Università di Milano, Ospedale Fatebenefratelli, Milano; Francesco Salinaro, Dept of Internal Medicine and Therapeutics, University of Pavia, Pavia, Italy, Emergency Department, Fondazione IRCCS Policlinico San Matteo, Pavia, Italy; Michelangelo Luciani, Department of Clinical and Molecular Medicine, Sapienza University, Rome, Italy; Cecilia Barnini, Department of Internal Medicine, University of Genoa and Policlinico San Martino, Genova, Italy; Stefania Morra di Cella, Department of Medical Sciences, University of Turin, Turin, Italy; Andrea Dalbeni, Department of Medicine, University of Verona, Verona, Italy; Simonetta Friso, Dept of Medicine, University of Verona and AOUI Verona, Policlinico GB Rossi, Verona, Italy; Michelangelo Luciani, Department of Clinical and Molecular Medicine, Sapienza University of Rome, Rome, Italy; Filippo Mearelli, Department of Internal Medicine, University of Trieste, Ospedale di Cattinara, Trieste, Italy; Paolo Malerba, Department of Clinical and Experimental Sciences, Clinica Medica, University of Brescia, Brescia, Italy; Francesco Cavallone, Department of Medical and Surgical Sciences, University of Foggia, Foggia, Italy; Damiano D'Ardes, Clinica Medica, Department of Medicine and Aging, "G. D’Annunzio" University of Chieti-Pescara, Chieti, Italy; Serena Notargiacomo, Department of Health, Life and Environmental Sciences, University of L'Aquila, L'Aquila, Italy; Alfredo De Giorgi, Department of Medical Sciences, University of Ferrara, Ferrara, Italy; Marta Mansi, San Carlo Hospital, Internal Medicine Department, Paderno Dugnano (MI), Italy; Elena Buzzetti, Department of Medical and Surgical Sciences for Children and Adults, University of Modena and Reggio Emilia, Italy; Andrea Ricci, Department of Medical and Surgical Sciences for Children and Adults, University of Modena and Reggio Emilia, Italy; Francesca Martelli, Italian Society of Internal Medicine (SIMI).

Elisabetta Tombolini, Thomas Teatini, Ernesto Crisafulli, Pier Paolo Sainaghi, Lorenzo Zileri Dal Verme, Salvatore Piano, Rebecca De Lorenzo, Gaetano Arcidiacono, Marco Podda, Luca Muratori, Claudia Gabiati, Francesco Salinaro, Michelangelo Luciani, Cecilia Barnini, Stefania Morra di Cella, Andrea Dalbeni, Simonetta Friso, Michelangelo Luciani, Filippo Mearelli, Paolo Malerba, Francesco Cavallone, Damiano D'Ardes, Serena Notargiacomo, Alfredo De Giorgi, Marta Mansi, Elena Buzzetti, Andrea Ricci, Francesca Martelli

Funding No funding was received for conduction this study.

\section{Declarations}

Conflict of interest The authors have no conflicts of interest to declare that are relevant to the content of this article.
Ethics approval The participating centers had the approval from the local ethical committee.

\section{References}

1. Wiersinga WJ, Rhodes A, Cheng AC et al (2020) Pathophysiology, transmission, diagnosis, and treatment of coronavirus disease 2019 (COVID-19): a review. JAMA 324(8):782-793. https://doi.org/10. 1001/jama.2020.12839

2. Onder G, Rezza G, Brusaferro S (2020) Case-fatality rate and characteristics of patients dying in relation to COVID-19 in Italy. JAMA 323(18):1775-1776. https://doi.org/10.1001/jama.2020.4683

3. Grippo F, Navarra S, Orsi C et al (2020) The role of COVID-19 in the death of SARS-CoV-2-positive patients: a study based on death certificates. J Clin Med. https://doi.org/10.3390/jcm9113459

4. Palmieri L, Vanacore N, Donfrancesco C et al (2020) Clinical characteristics of hospitalized individuals dying with COVID-19 by age group in Italy. J Gerontol A Biol Sci Med Sci 75(9):1796-1800. https://doi.org/10.1093/gerona/glaa146

5. Lenti MV, Corazza GR, Di Sabatino A (2020) Carving out a place for internal medicine during COVID-19 epidemic in Italy. J Intern Med 288(2):263-265. https://doi.org/10.1111/joim.13079

6. Montagnani A, Pieralli F, Gnerre P et al (2020) COVID-19 pandemic and internal medicine units in Italy: a precious effort on the front line. Intern Emerg Med 15(8):1595-1597. https://doi.org/10. 1007/s11739-020-02454-5

7. Gupta S, Hayek SS, Wang W et al (2020) Factors associated with death in critically ill patients with coronavirus disease 2019 in the US. JAMA Intern Med. https://doi.org/10.1001/jamainternmed. 2020.3596

8. Grasselli G, Tonetti T, Protti A et al (2020) Pathophysiology of COVID-19-associated acute respiratory distress syndrome: a multicentre prospective observational study. Lancet Respir Med 8(12):1201-1208. https://doi.org/10.1016/S2213-2600(20)30370-2

9. Guaraldi G, Meschiari M, Cozzi-Lepri A et al (2020) Tocilizumab in patients with severe COVID-19: a retrospective cohort study. Lancet Rheumatol 2(8):e474-e484. https://doi.org/10.1016/S26659913(20)30173-9

10. Soria A, Galimberti S, Lapadula G et al (2021) The high volume of patients admitted during the SARS-CoV-2 pandemic has an independent harmful impact on in-hospital mortality from COVID-19. PLoS ONE 16(1):e0246170. https://doi.org/10.1371/journal.pone. 0246170

11. Albani F, Fusina F, Granato E et al (2021) Corticosteroid treatment has no effect on hospital mortality in COVID-19 patients. Sci Rep 11(1):1015. https://doi.org/10.1038/s41598-020-80654-x

12. Richardson S, Hirsch JS, Narasimhan M et al (2020) Presenting characteristics, comorbidities, and outcomes among 5700 patients hospitalized with COVID-19 in the New York city area. JAMA 323(20):2052-2059. https://doi.org/10.1001/jama.2020.6775

13. Garibaldi BT, Fiksel J, Muschelli J et al (2021) Patient trajectories among persons hospitalized for COVID-19: a cohort study. Ann Intern Med 174(1):33-41. https://doi.org/10.7326/M20-3905

14. Wongvibulsin S, Garibaldi BT, Antar AAR et al (2021) Development of severe COVID-19 adaptive risk predictor (SCARP), a calculator to predict severe disease or death in hospitalized patients with COVID-19. Ann Intern Med. https://doi.org/10.7326/M20-6754

15. Bahat $\mathrm{G}$ (2020) Covid-19 and the renin angiotensin system: implications for the older adults. J Nutr Health Aging 24(7):699-704. https://doi.org/10.1007/s12603-020-1403-7

16. Behl T, Kaur I, Bungau $S$ et al (2020) The dual impact of ACE2 in COVID-19 and ironical actions in geriatrics and pediatrics with possible therapeutic solutions. Life Sci 257:118075. https://doi.org/ 10.1016/j.lfs.2020.118075 
17. Mancia G, Rea F, Ludergnani M et al (2020) Renin-angiotensinaldosterone system blockers and the risk of Covid-19. N Engl J Med 382(25):2431-2440. https://doi.org/10.1056/NEJMoa2006923

18. Nunez-Gil IJ, Fernandez-Perez C, Estrada V et al (2020) Mortality risk assessment in Spain and Italy, insights of the HOPE COVID-19 registry. Intern Emerg Med. https://doi.org/10.1007/ s11739-020-02543-5

19. Group RC, Horby P, Lim WS et al (2021) Dexamethasone in hospitalized patients with Covid-19. N Engl J Med 384(8):693-704

20. Salama C, Han J, Yau L et al (2021) Tocilizumab in patients hospitalized with Covid-19 pneumonia. N Engl J Med 384(1):20-30. https://doi.org/10.1056/NEJMoa2030340

21. Ferreyro BL, Angriman F, Scales DC (2020) Noninvasive oxygenation strategies for acute hypoxemic respiratory failure-reply. JAMA 324(18):1906-1907. https://doi.org/10.1001/jama.2020.18602
22. Bellani G, Grasselli G, Cecconi M et al (2021) Noninvasive ventilatory support of COVID-19 patients outside the intensive care units (WARd-COVID). Ann Am Thorac Soc. https://doi.org/10.1513/ AnnalsATS.202008-10800C

23. Di Domenico SL, Coen D, Bergamaschi M et al (2020) Clinical characteristics and respiratory support of 310 COVID-19 patients, diagnosed at the emergency room: a single-center retrospective study. Intern Emerg Med. https://doi.org/10.1007/s11739-020-02548-0

24. Ageno W, Cogliati C, Perego M et al (2021) Clinical risk scores for the early prediction of severe outcomes in patients hospitalized for COVID-19. Intern Emerg Med. https://doi.org/10.1007/ s11739-020-02617-4

Publisher's Note Springer Nature remains neutral with regard to jurisdictional claims in published maps and institutional affiliations.

\section{Authors and Affiliations}

\section{Elena Corradini ${ }^{1} \cdot$ Paolo Ventura $^{1} \cdot$ Walter Ageno $^{2}$. Chiara Beatrice Cogliati ${ }^{3} \cdot$ Maria Lorenza Muiesan $^{4}$. Domenico Girelli ${ }^{5}$. Mario Pirisi ${ }^{6}$. Antonio Gasbarrini ${ }^{7} \cdot$ Paolo Angeli $^{8}$. Patrizia Rovere Querini ${ }^{9} \cdot$ Emanuele Bosi $^{9}$. Moreno Tresoldi $^{9} \cdot$ Roberto Vettor $^{10}$. Marco Cattaneo ${ }^{11}$. Fabio Piscaglia ${ }^{12}$. Antonio Luca Brucato ${ }^{13}$. Stefano Perlini ${ }^{14}$. Paolo Martelletti ${ }^{15} \cdot$ Roberto Pontremoli $^{16}$. Massimo Porta ${ }^{17}$. Pietro Minuz ${ }^{18}$. Oliviero Olivieri ${ }^{19}$. Giorgio Sesti ${ }^{20}$. Gianni Biolo ${ }^{21}$. Damiano Rizzoni ${ }^{22}$. Gaetano Serviddio ${ }^{23}$. Francesco Cipollone ${ }^{24}$. Davide Grassi ${ }^{25}$. Roberto Manfredini ${ }^{26} \cdot$ Guido Luigi Moreo $^{27} \cdot$ Antonello Pietrangelo $^{1}$ (1) $\cdot$ SIMI-COVID-19 Collaborators}

1 Unit of Internal Medicine, University Hospital Policlinico di Modena, Modena, Italy

2 Department of Medicine and Surgery, University of Insubria, Varese, Italy

3 Department of Internal Medicine, Luigi Sacco Hospital, University of Milan, Milan, Italy

4 Department of Clinical and Experimental Sciences, University of Brescia-Medicina 2, ASST Spedali Civili Brescia, Brescia, Italy

5 Department of Medicine, Section of Internal Medicine, University of Verona, Verona, Italy

6 Internal Medicine Department of Translational Medicine, University of Piemonte Orientale, Novara, Italy

7 Department of Medical and Surgical Sciences, Fondazione Policlinico Gemelli IRCCS, Università Cattolica del S. Cuore, Rome, Italy

8 Unit of Internal Medicine and Hepatology (UIMH), Department of Medicine-DIMED, University of Padova, Padua, Italy

9 Unit of Internal Medicine, IRCCS Ospedale San Raffaele, Vita-Salute San Raffaele University, Milan, Italy

10 Department of Medicine, Università degli Studi di Padova, Padua, Italy

11 Dipartimento di Scienze della Salute, Università degli Studi di Milano, Milan, Italy

12 Division of Internal Medicine, IRCCS Azienda Ospedaliero, Universitaria di Bologna, Bologna, Italy

13 Department of Internal Medicine, Fatebenefratelli, Milan, Italy
14 Emergency Department, Fondazione IRCCS Policlinico San Matteo, Pavia, Italy

15 Department of Clinical and Molecular Medicine, Sapienza University, Rome, Italy

16 Department of Internal Medicine, University of Genoa and Policlinico San Martino, Genoa, Italy

17 Department of Medical Sciences, University of Turin, Turin, Italy

18 Department of Internal Medicine, University of Verona, Verona, Italy

19 Department of Medicine, Policlinico GB Rossi, University of Verona and AOUI Verona, Verona, Italy

20 Department of Clinical and Molecular Medicine, Sapienza University of Rome, Rome, Italy

21 Department of Internal Medicine, Ospedale di Cattinara, University of Trieste, Trieste, Italy

22 Department of Clinical and Experimental Sciences, Clinica Medica, University of Brescia, Brescia, Italy

23 Department of Medical and Surgical Sciences, University of Foggia, Foggia, Italy

24 Department of Medicine and Aging, Clinica Medica, "G. D’Annunzio" University of Chieti-Pescara, Chieti, Italy

25 Department of Health, Life and Environmental Sciences, University of L'Aquila, L'Aquila, Italy

26 Department of Medical Sciences, University of Ferrara, Ferrara, Italy

27 Internal Medicine Department, San Carlo Hospital, Paderno Dugnano, Milan, Italy 